\title{
Fault Diagnosis Technology of Plunger Pump based on EMMD-Teager
}

\author{
Shijie Deng", Liwei Tang, Xujun Su, and Jinli Che \\ Department of Artillery Engineering, Army Engineering University, Shijiazhuang, 050003, China
}

\begin{abstract}
Based on the analysis of common failure modes of plunger pumps, a fault diagnosis method based on EMMD decomposition and Teager energy operator demodulation is proposed to solve the problem of weak characteristic signals in early failure of plunger pumps. Firstly, the extremum field mean mode decomposition (EMMD) is used to obtain the finite mode component IMF and the residual C. Then, the IMF component is demodulated by Teager energy operator, and the characteristic peak appears in the spectrum. The energy information of the feature frequency points is extracted to form the feature vectors, which can be used as the proportion. The elements in the vectors are screened by the classification sensitivity, and the effective feature vectors are finally obtained. The experimental results show that the EMMD-Teager method can filter the signal effectively and extract features from the frequency domain conveniently. The selected feature vectors can accurately classify the three states of the normal plunger pump, plunger hole wear, and slipper wear.
\end{abstract}

Keywords: Teager energy operator; EMMD decomposition; plunger pump; fault diagnosis

(Submitted on March 27, 2019; Revised on April 26, 2019; Accepted on June 23, 2019)

(C) 2019 Totem Publisher, Inc. All rights reserved.

\section{Introduction}

In the process of rocket artillery adjustment, the key to ensure rapid and accurate gun adjustment is the stable operation of plunger pumps. If the failure of a plunger pump results in uneven output and insufficient pressure, it will seriously affect the rapid response ability and shooting accuracy of weapon equipment. S-shaped gun adjustment, flutter, wheezing, and other phenomena occur, and the guns cannot be adjusted. This will cause irreparable military losses and even threaten the lives of soldiers on the battlefield. Therefore, it is necessary to study the fault diagnosis of plunger pumps.

When a plunger pump fails, there will be a periodic instantaneous impact along with the rotation of the motor. The impact signal is transmitted to the pump shell through the bearing. Therefore, the fault can be diagnosed by collecting and analyzing the vibration signal of the pump shell. However, the vibration of the motor will drive the resonance of a large number of equipment around it and increase the source of vibration, which will cause the measured vibration signal of the plunger pump to be submerged in a large amount of noise. Traditional Fourier transform cannot extract useful features, and envelope spectrum analysis also needs to improve the resonance response signal of the pump by filtering based on prior knowledge. Therefore, determining how to filter and select the frequency band effectively is the key to fault diagnosis.

Empirical mode decomposition (EMD) is a new signal processing method that was proposed by Huang [1] in 1998, and it is used to process non-linear and unsteady signals. EMD can adaptively decompose signals into different frequency bands. Some scholars have made improvements on the basis of EMD [2-3]. Combining EMD with the adaptive time-varying filter (ATVFD), Gai [4] proposed a new decomposition method named extremum field mean mode decomposition (EMMD). Compared with EMD, EMMD improves the solution of the mean curve. It uses the median theorem to obtain the local mean so that only one spline interpolation is used for each iteration. The mean curve is obtained more accurately, which improves the decomposition efficiency.

The Teager energy operator (TEO) is a non-linear operator that was proposed by Kaiser [5]. It estimates the total energy required by the signal source to generate dynamic signals through the non-linear combination of the instantaneous value of

\footnotetext{
* Corresponding author.

E-mail address: dsj_sjz@163.com
} 
the signal and signal differentiation. It can enhance the transient characteristics of the signal and is suitable for detecting the impulse components in the signal. It can also further obtain the envelope and instantaneous frequency of the signal. This method has low computational complexity, high efficiency, good time resolution, and good adaptive ability to the instantaneous changes of signals [6-7]. In this paper, the fault diagnosis of plunger pumps based on EMMD decomposition and Teager energy operator demodulation is carried out in order to distinguish the plunger pumps in different states.

\section{EMMD Decomposition and Teager Energy Operator}

\subsection{EMMD Decomposition}

Compared with the traditional EMD decomposition method, EMMD has been improved in calculating the local mean. The EMD decomposition first finds the upper and lower extremum points of the signal, then finds the upper and lower envelopes respectively by spline interpolation, and then finds the local mean by adding the upper and lower envelopes. EMMD is based on the mean value theorem of the definite integral. The local mean is obtained by averaging all the data between the three adjacent extreme points as the mean of the location of the intermediate extreme point. Then, the obtained local mean sequence is fitted by spline interpolation, and the mean curve is obtained. Therefore, the EMMD method only needs one spline interpolation in the process of finding the mean curve each time. The specific steps of EMMD decomposition are as follows:

(1) Initialization: $i=1, c_{i}(t)=x(t), c_{i}(t)$ represents a sequence of margins;

(2) Initialization: $h_{i}(t)=c_{i}(t)$;

(3) Find all local extreme points of $h_{i}(t)$ (no distinction between maxima and minima), and form a sequence of extreme points $p(k)$;

(4) According to the interval length of three adjacent extremum points and the integration of data, the local mean of the intermediate extremum points is obtained by using the definite integral mean value theorem:

$$
g(k)=\frac{p(k)-p(k-1)}{p(k+1)-p(k-1)} g^{(k-1, k)}+\frac{p(k+1)-p(k)}{p(k+1)-p(k-1)} g^{(k, k+1)}
$$

Where $k$ denotes the $k^{\text {th }}$ extremum point, $p k$ denotes the position of the $k^{\text {th }}$ extremum point in the original signal, and $g^{(k, k+1)}$ denotes the mean of all data between the $k^{\text {th }}$ extremum point and the $k+1$ extremum point.

(5) The spline interpolation function is used to fit and extend the local mean sequence $g(k)$, and the local mean curve $m_{i}(t)$ is obtained.

(6) First assigning sequence $h_{i}(t)$ to a temporary sequence $\operatorname{preh}_{i}(t)$ and then subtracting local mean curve $m_{i}(t)$ from it, a new sequence with low frequency can be obtained:

$$
\sum_{t=0}^{N} \frac{\left|h_{i}(t)-\operatorname{preh}_{i}(t)\right|^{2}}{\operatorname{preh}_{i}(t)^{2}} \leq \Delta_{S D}
$$

(7) For $\Delta_{S D}$, generally choose 0.2 to 0.3 . If the condition is satisfied, the $i^{\text {th }}$ IMF component $i m f_{i}(t)=h_{i}(t)$ is decomposed and a new residual sequence $c_{i+1}=c_{i}-i m f_{i}(t)$ is obtained. If not, step (3) is returned until it is satisfied.

(8) If there are no fewer than two extreme points of $c_{i+1}$, then $i=i+1$, and return to step (2); otherwise, the decomposition ends, and $c_{i+1}$ is a sequence of margins.

As an improvement of EMD, EMMD inherits the ability of EMD to deal with non-linear and unsteady signals. 
However, the traditional EMD only uses the extreme point information of the data, ignoring the non-extreme points, while EMMD uses all the data between the extreme points when calculating the mean curve. EMMD can eliminate the DC component hidden in the local data, which is more in line with the conditions of the intrinsic mode function. Additionally, the number of iterations of EMMD is less than that of EMD, so the decomposition efficiency is higher [8-10].

\subsection{Teager Energy Operator}

The definition of the Teager energy operator is as follows:

$$
T[x(t)]=\dot{x}(t)^{2}-x(t) \ddot{x}(t)
$$

Where $x(t)$ denotes a one-dimensional signal and $\dot{x}(t)$ and $\ddot{x}(t)$ denote the first and second derivatives of the signal, respectively.

Suppose the one-dimensional signal is a sinusoidal signal:

$$
\mathrm{x}(t)=A(t) \sin (\omega t+\varphi)
$$

By calculating the energy operator, the output is the product of the square of the instantaneous amplitude and the square of the instantaneous frequency of the vibration, such as in Equation (4). Compared with the traditional expression of energy amplitude squared, it multiplies the instantaneous frequency squared. Because of the high frequency of the shock signal, the Teager operator can effectively enhance the impulse component.

$$
T[x(t)]=A(t)^{2} \omega^{2}
$$

The Teager operator of discrete signals is implemented as follows:

$$
T[x(k)]=x(k)^{2}-x(k-1) x(k+1)
$$

\section{Analysis of Measured Signal of Plunger Pump}

\subsection{Experimental Equipment}

When the plunger pump runs in the fault state, the vibration signal of the pump body is composed of several frequency family components, where each component can be regarded as the amplitude modulation signal. The original signal is decomposed into frequency families with different center frequencies using the EMMD method. The Teager energy operator is used to demodulate each frequency family, and the final spectrum is obtained to extract fault features.

In this paper, the vibration signal of the plunger pump is collected from the hydraulic pump test-bed, as shown in Figure 1(a). The plunger pump model is the 25SCY14-1B axial swashplate plunger pump with seven plungers. The motor model is the YVF2-160M-4 variable frequency speed three-phase asynchronous motor. The model of the acceleration vibration sensor is CA-YD-182-10. The installation position is shown in Figure 1(b). The plunger moves axially relative to the cylinder block, and the axial vibration will have a larger value, so the sensor is placed on the end face. When the outlet pressure of the pump is $14 \mathrm{MPa}$ and the motor speed is $1500 \mathrm{r} / \mathrm{min}$, the corresponding shaft frequency is $25 \mathrm{~Hz}$, the plunger frequency is $175 \mathrm{~Hz}$ (seven times the shaft frequency), and the sampling frequency is set to $10 \mathrm{kHz}$. In the experiment, diagnostic analysis is carried out on three states of the normal plunger pump, plunger hole wear, and slipper wear.

\subsection{Default Failure}

The fault element in the experiment is the preset fault. Firstly, the axial vibration signal at the end cap is collected by the normal piston pump experiment. Then, the normal parts are replaced by the fault parts. The wear of the plunger hole and slipper of the piston pump is simulated artificially, as shown in Figure 2. In the experiment, a groove is drawn on the inner wall of a plunger hole to imitate the wear state of the plunger hole. The groove depth is $0.1 \mathrm{~mm}$, and the width is $0.4 \mathrm{~mm}$. Then, the slipper wear is simulated by grinding and polishing the surface of the slipper, and the wear depth is $0.4 \mathrm{~mm}$. 


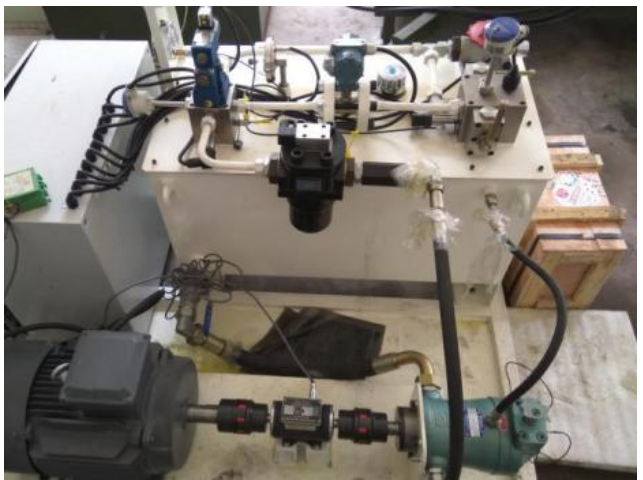

(a)

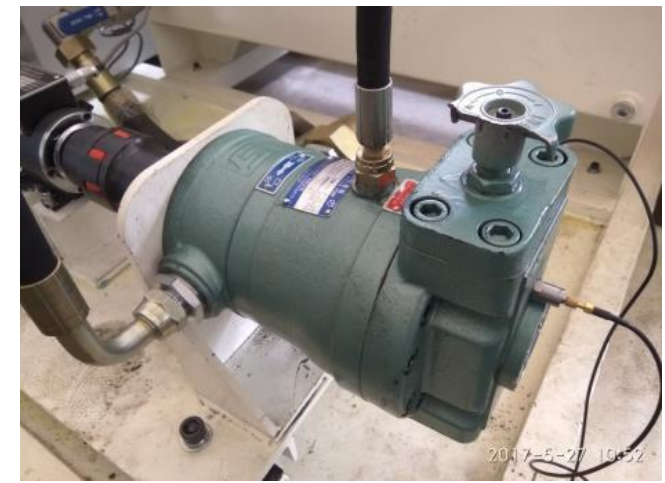

(b)

Figure 1. Experiment rig of plunger pump and installation position of vibration sensor

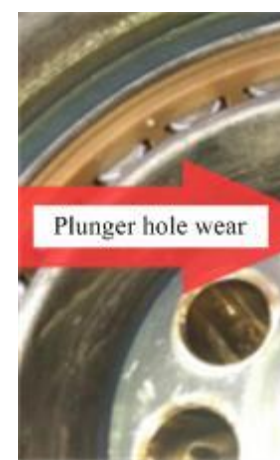

(a)

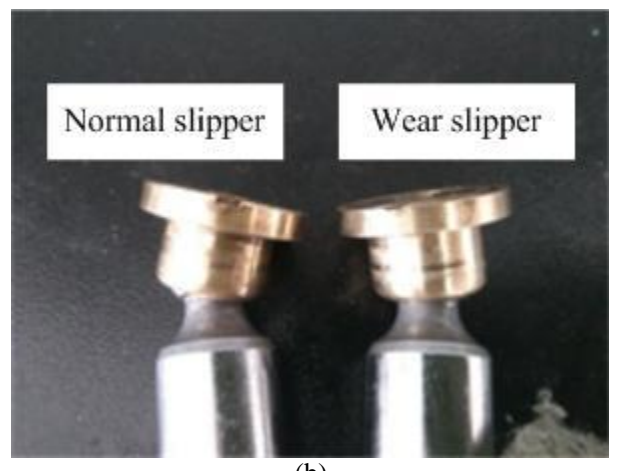

(b)

Figure 2. Plunger hole wear and slippage wear

\subsection{Fault Diagnosis}

In the experiment, the vibration signals of three states are collected for ten seconds, and one second of each state is randomly selected for analysis. The time and frequency domains of the three states are shown in Figure 3.
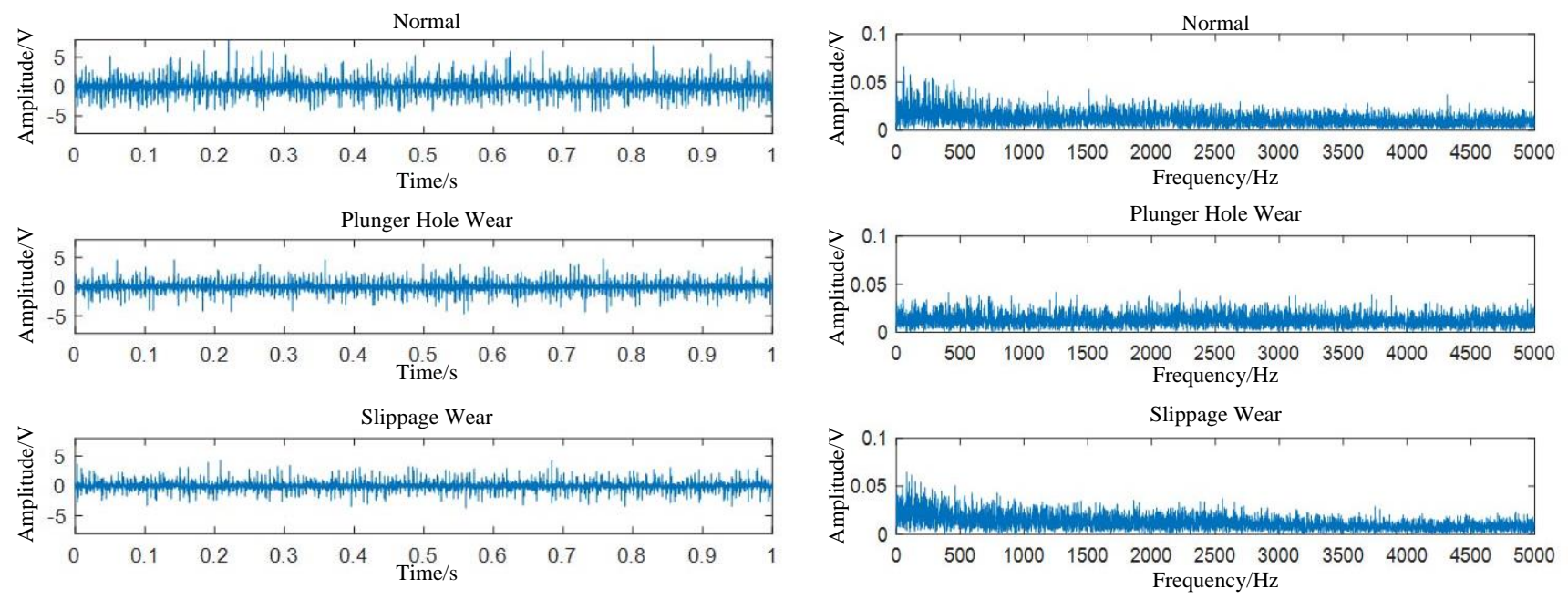

(a) Time-domain

(b) Frequency-domain

Figure 3. Signals in various states

It is difficult to sum up the differences between different states in the unprocessed time-domain waveforms and frequency-domain waveforms. In order to extract the fault characteristics of plunger pumps, the signal of each state is decomposed into extremum field mean mode decomposition (EMMD). Because the impulse signal is mainly distributed in the high frequency part, only the original signal is decomposed by third-order EMMD. The third-order IMF components and margins under three states are shown in Figure 4. 


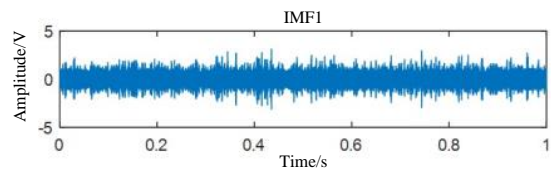

IMF2

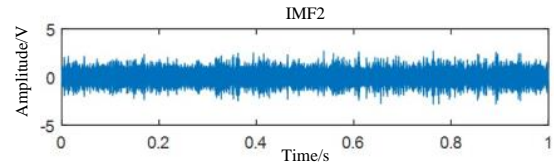

IMF3
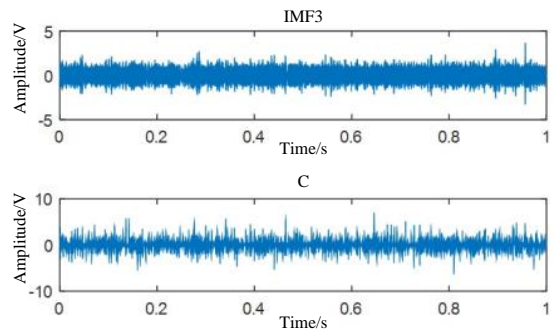

(a) Normal State

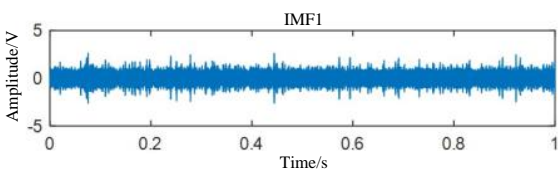

IMF2

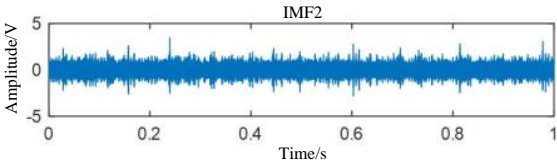

IMF
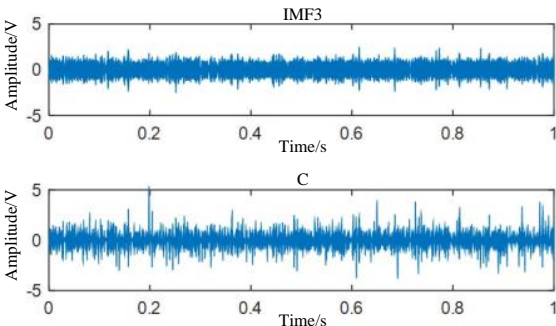

(b)Plunger Hole Wear
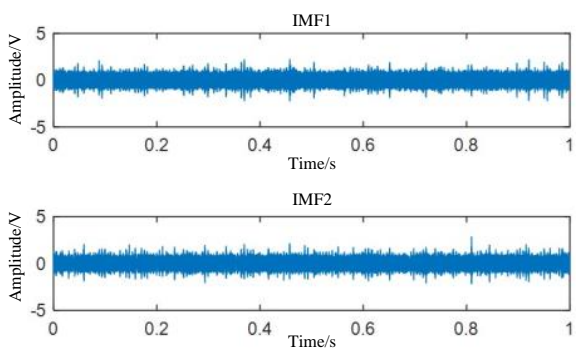

IMF3
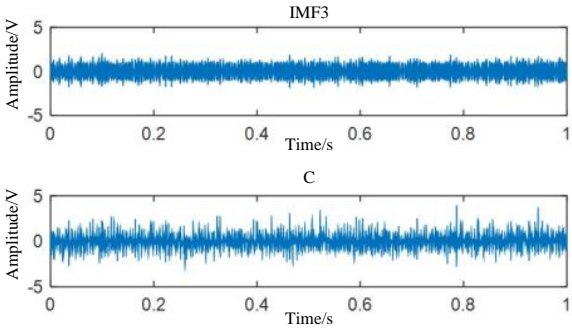

(c)Slipper Wear

Figure 4. Third-order IMF and allowance $\mathrm{C}$ in three states

The Teager energy operator is used to demodulate the obtained components and margins, and Fourier transform is used to obtain the Teager spectrum, as shown in Figure 5. From Figure 5(a), it can be seen that there are peaks at $171 \mathrm{~Hz}$ (plunger frequency, seven-fold frequency), $341 \mathrm{~Hz}, 512 \mathrm{~Hz}$ (two-fold and three-fold plunger frequency) of all components and margins of normal signals, and $23 \mathrm{~Hz}$ (axis frequency) of IMF 3. From Figure 5(b), it can be seen that the Teager spectrum of plunger hole wear appears obvious at $23 \mathrm{~Hz}$ (axis frequency) on the basis of the normal signal spectrum, and there is a side frequency of $23 \mathrm{~Hz}$ (axis frequency) offset to both sides at $171 \mathrm{~Hz}$ (plunger frequency). Figure 5 (c) shows that only 171 of each component and two, three, and four times of the plunger frequency have no more peaks. This is because the vibration signal between the slipper and the swashplate is broadband, the energy in the frequency domain is more dispersed, and the four times $(682 \mathrm{~Hz})$ of the plunger frequency disappears compared with the normal axis frequency. Therefore, fault diagnosis can be carried out by extracting the characteristics of corresponding frequency points from the first three IMF components and the remaining Teager spectrum of the plunger pump vibration signal.
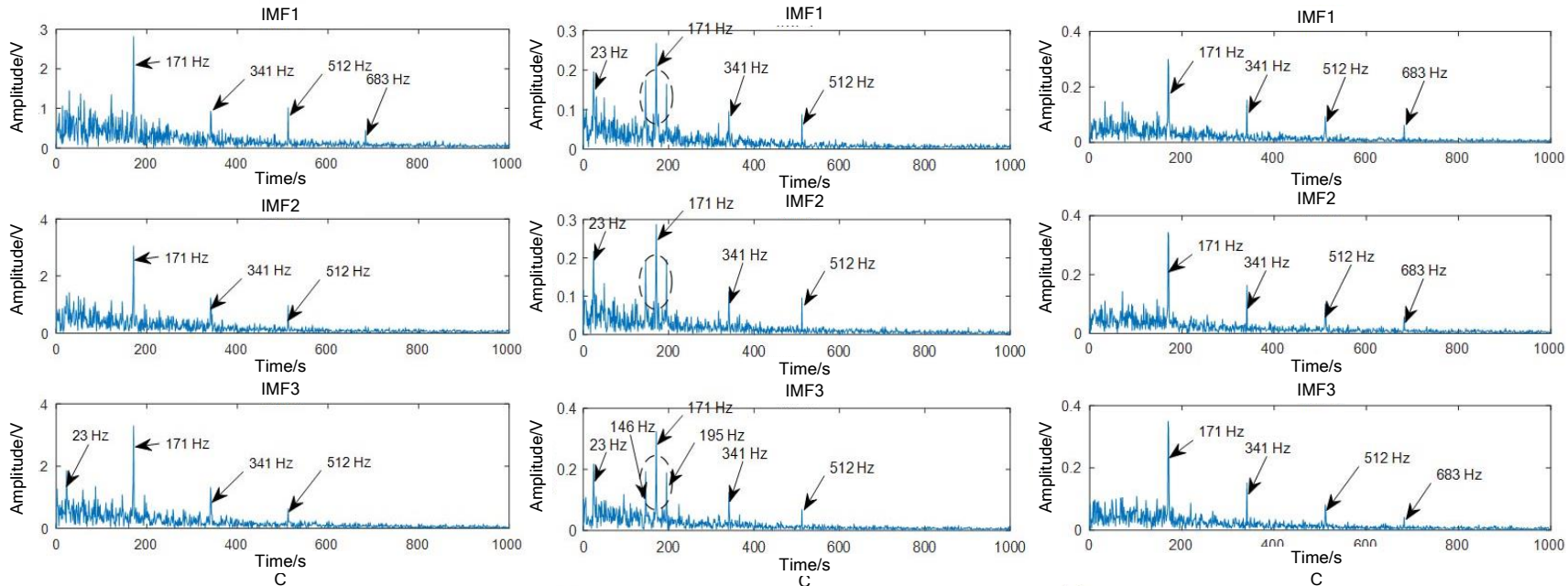

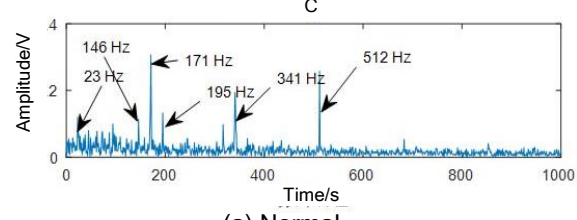

(a) Normal

State

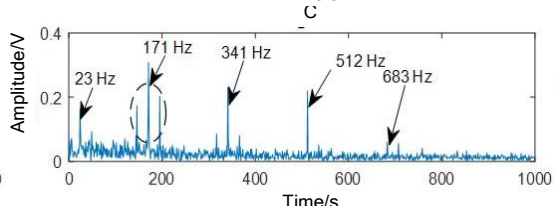

(b) Plunger Hole

Wear

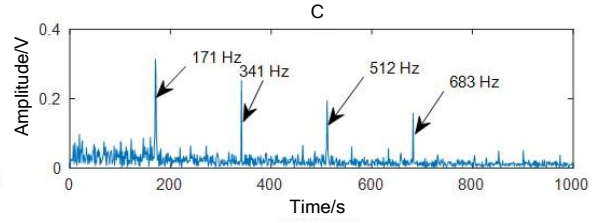

(c) Slipper Wear

Figure 5. Teager spectra of components 
In this paper, the energy proportion of each frequency point $(23 \mathrm{~Hz}, 146 \mathrm{~Hz}, 171 \mathrm{~Hz}, 195 \mathrm{~Hz}, 341 \mathrm{~Hz}, 512 \mathrm{~Hz}, 683 \mathrm{~Hz})$ is simply calculated as the eigenvector of the signal, as shown in Table 1(a) to (d).

Table 1. (a) Energy proportion of IMF1 frequency points
\begin{tabular}{|c|c|c|c|c|c|c|c|}
\hline \multirow{2}{*}{ State } & \multicolumn{7}{|c|}{ IMF1 } \\
\cline { 2 - 8 } & T1 & T2 & T3 & T4 & T5 & T6 & T7 \\
\hline A & 0.171 & 0.444 & 0.111 & 0.101 & 0.098 & 0.061 & 0.014 \\
\hline B & 0.179 & 0.477 & 0.168 & 0.084 & 0.048 & 0.042 & 0.003 \\
\hline C & 0.076 & 0.730 & 0.042 & 0.032 & 0.081 & 0.028 & 0.010 \\
\hline
\end{tabular}

Table 1. (b) Energy proportion of IMF2 frequency points

\begin{tabular}{|c|c|c|c|c|c|c|c|}
\hline \multirow{2}{*}{ State } & \multicolumn{7}{|c|}{ IMF2 } \\
\cline { 2 - 8 } & T8 & T9 & T10 & T11 & T12 & T13 & T14 \\
\hline A & 0.198 & 0.536 & 0.036 & 0.032 & 0.159 & 0.038 & 0.002 \\
\hline B & 0.118 & 0.559 & 0.143 & 0.068 & 0.072 & 0.038 & 0.001 \\
\hline C & 0.095 & 0.678 & 0.050 & 0.032 & 0.095 & 0.042 & 0.009 \\
\hline
\end{tabular}

Table 1. (c) Energy proportion of IMF3 frequency points
\begin{tabular}{|c|c|c|c|c|c|c|c|}
\hline \multirow{2}{*}{ State } & \multicolumn{7}{|c|}{ IMF3 } \\
\cline { 2 - 8 } & T15 & T16 & T17 & T18 & T19 & T20 & T21 \\
\hline A & 0.126 & 0.633 & 0.047 & 0.046 & 0.126 & 0.020 & 0.002 \\
\hline B & 0.280 & 0.437 & 0.124 & 0.085 & 0.058 & 0.014 & 0.001 \\
\hline C & 0.060 & 0.747 & 0.043 & 0.044 & 0.070 & 0.030 & 0.007 \\
\hline
\end{tabular}

Table 1. (d) Energy proportion of each frequency point of residual C

\begin{tabular}{|c|c|c|c|c|c|c|c|}
\hline \multirow{2}{*}{ State } & \multicolumn{7}{|c|}{ C } \\
\cline { 2 - 8 } & T22 & T23 & T24 & T25 & T26 & T27 & T28 \\
\hline A & 0.087 & 0.394 & 0.042 & 0.040 & 0.241 & 0.182 & 0.014 \\
\hline B & 0.161 & 0.346 & 0.111 & 0.105 & 0.151 & 0.120 & 0.007 \\
\hline C & 0.030 & 0.500 & 0.027 & 0.005 & 0.182 & 0.163 & 0.093 \\
\hline
\end{tabular}

Note:

(1) A indicates the normal condition, B indicates the wear of the plunger hole, and C indicates the wear of the slipper;

(2) T1-T7 represent the $23 \mathrm{~Hz}, 146 \mathrm{~Hz}, 171 \mathrm{~Hz}, 195 \mathrm{~Hz}, 341 \mathrm{~Hz}, 512 \mathrm{~Hz}$, and $683 \mathrm{~Hz}$ frequency points of IMF1, respectively, and T8-T28 represent the frequency points of other components, respectively.

The classification sensitivity of each feature is calculated by synthesizing multiple samples to judge the ability of a frequency point to classify faults [11-12], as shown in Table 2.

Table 2. Classification sensitivity corresponding to each feature point

\begin{tabular}{|l|c|c|c|c|c|c|}
\hline \multicolumn{7}{|c|}{ IMF1 } \\
\hline 2.75 & $\mathbf{4 . 8 0}$ & 2.17 & 2.60 & 2.85 & 2.05 & 2.96 \\
\hline \multicolumn{7}{|c|}{ IMF2 } \\
\hline 2.59 & 3.22 & 2.09 & 2.49 & 4.22 & 2.41 & $\mathbf{4 . 2 7}$ \\
\hline 3.08 & 4.09 & 1.88 & 2.83 & 2.18 & 2.40 & 2.49 \\
\hline \multicolumn{7}{|c|}{ IMF3 } \\
\hline 2.68 & $\mathbf{5 . 8 9}$ & 3.34 & $\mathbf{5 . 0 6}$ & 2.89 & 2.53 & $\mathbf{8 . 2 8}$ \\
\hline
\end{tabular}

The formula of classification sensitivity is as follows:

$$
\begin{aligned}
& \eta_{k}=\frac{J_{k}}{S_{k}}, \quad k=1,2, \cdots, m \\
& J_{k}=\frac{1}{C(C-1)} \sum_{a=1}^{C} \sum_{b=1}^{C} D_{k}^{a b}
\end{aligned}
$$




$$
S_{k}=\frac{1}{C} \sum_{a=1}^{C}\left[\frac{1}{N(N-1)} \sum_{i, j=1}^{N} D\left(x_{i}^{a}, x_{j}^{a}\right)\right]
$$

The formulas for $\eta_{k}, J_{k}$, and $S_{k}$ represent the sensitivity of classification, the distance between classes, and the distance within classes, respectively; $x_{i}^{a}$ denotes an eigenvalue of class $a$ samples under the $k^{\text {th }}$ feature; $D\left(\mathrm{x}_{i}{ }^{a}, x_{j}^{a}\right)$ denotes the distance between two samples of the same kind; $D_{k}^{a b}$ denotes the average distance between two samples of different classes; $N$ denotes the number of samples of class $a$; and $C$ denotes the number of classes.

The data in Table 1 represent the ratio of energy at each frequency point to the sum of energy at all selected frequency points under each IMF component. Five groups of 15 samples are randomly sampled from each state of the collected vibration signals. According to Equations (6) to (8), the classification sensitivity corresponding to each feature frequency point is obtained, and the classification sensitivity is sorted in descending order. The greater the sensitivity, the more different categories can be distinguished under this feature. The five features with the greatest sensitivity are selected to form a new feature vector, as shown in Table 3.

\begin{tabular}{|c|c|c|c|c|c|}
\multicolumn{7}{|c|}{ Table 3. New eigenvector } \\
\hline State & T28 & T23 & T25 & T2 & T14 \\
\hline A & 0.014 & 0.394 & 0.040 & 0.444 & 0.002 \\
\hline B & 0.007 & 0.346 & 0.105 & 0.477 & 0.001 \\
\hline C & 0.093 & 0.500 & 0.005 & 0.730 & 0.009 \\
\hline
\end{tabular}

In order to clearly express the classification effect of the selected features, the energy proportion corresponding to the 15 samples based on the new feature vector is normalized and drawn in the distribution chart, as shown in Figure 6.

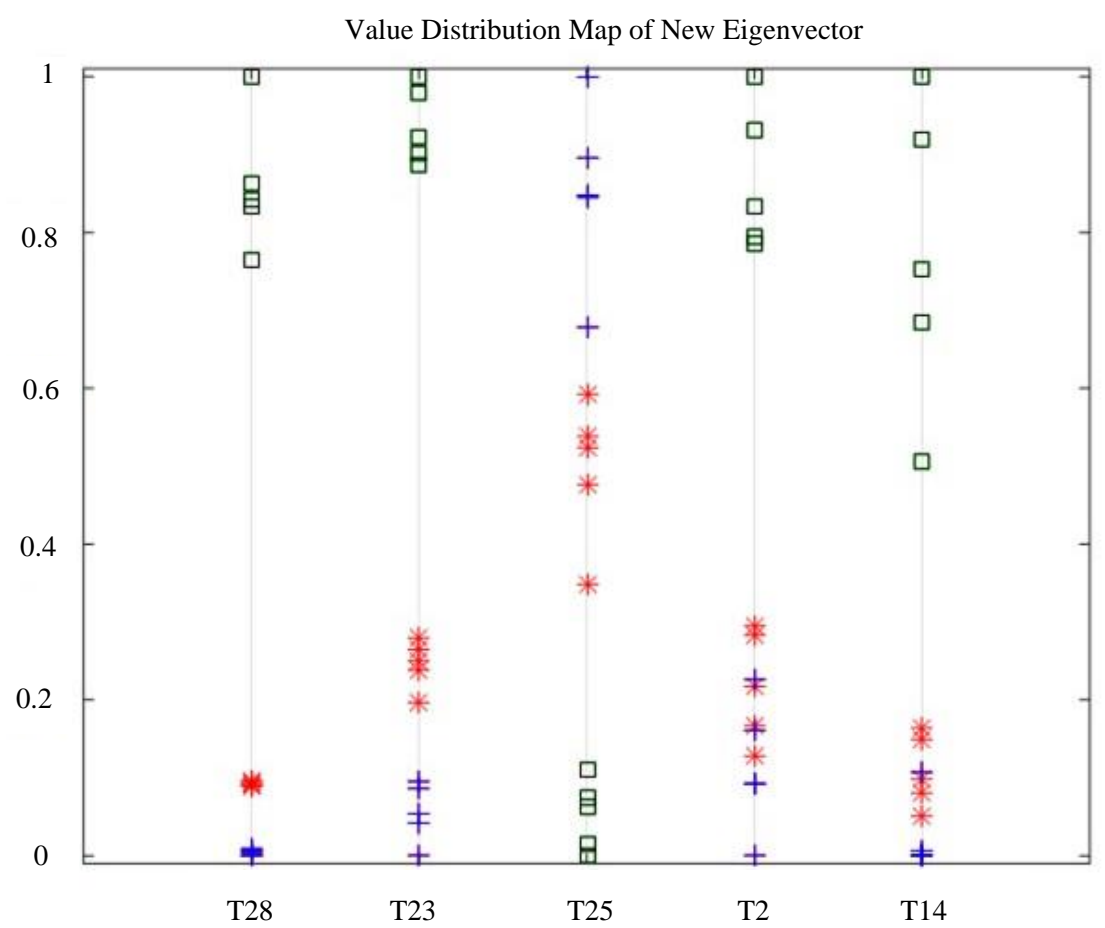

Figure 6. Distribution of five eigenvalue domains with the highest sensitivity

The abscissa in the graph is the five features sorted from largest to smallest according to the sensitivity, and the ordinate is the maximum and minimum normalized energy proportion. "*" stands for the normal condition, "+" for the wear of the plunger hole, and " $\square$ " for the wear of the slippers. It can be seen from the figure that the three states are clearly distinguished under the highest classification sensitivity of T28 (683HZ frequency point of residual C), the second highest T23 (146HZ frequency point of residual C), and T25 (171HZ frequency point of residual C). The lower sensitivity of T2 and T14 can only distinguish slipper wear, but the normal state and plunger hole wear cannot be distinguished. 


\section{Conclusions}

It is very difficult to extract fault features of plunger pumps when the early fault signal is weak and completely covered by background noise. Vibration signals are processed by combining EMMD decomposition and the Teager energy operator, which can effectively extract frequency features from the frequency domain.

By extracting the energy proportion of characteristic frequency points from the vibration signal after processing, the feature vectors are formed, and the feature vectors are screened through classification sensitivity. The filtered feature vectors can effectively distinguish the normal states of the plunger pump, plunger hole wear, and slipper wear.

\section{Acknowledgements}

We would like to thank the reviewers for their valuable comments. This work was partially funded by the National Natural Science Foundation of China and the Military Research Foundation of China.

\section{References}

1. N. E. Huang, S. Zheng, S. R. Long, M. C. Wu, H. H. Shih, Q. N. Zheng, et al., "The Empirical Mode Decomposition and the Hilbert Spectrum for Nonlinear and Nonstationary Time Series Analysis," Proceedings of the Royal of London Series A, Vol. 454, No. 1971, pp. 903-995, 1998

2. Z. Q. Ma, Y. C. Li, Z. Liu, and C. J. Gu, "Rolling Bearing's Fault Feature Extraction based on Variational Mode Decomposition and Teager Energy Operator," Journal of Vibration and Shock, Vol. 35, No. 13, pp. 134-139, 2016

3. Z. Wu and N. E. Huang, "Ensemble Empirical Mode Decomposition: A Noise-Assisted Data Analysis Method," Advances in Adaptive Data Analysis, Vol. 1, No. 1, pp. 26-31, 2011

4. Q. Gai, "Theoretical Research and Application of Time-Frequency Analysis of Local Waves," Dalian University of Technology, 2001

5. J. F. Kaiser, "On a Simple Algorithm to Calculate the Energy' of a Signal," in Proceedings of International Conference on Acoustics, Speech, and Signal Processing, Vol. 1, pp. 381-384, IEEE, 1990

6. Q. L. Yang, J. M. Mei, J. Xiao, L. L. Zhang, and Y. K. Xiao, "Weak Fault Feature Extraction for Bearings based on an Order Cepstrum Enhanced with Teager Energy Operator," Journal of Vibration and Shock, Vol. 34, No. 6, pp. 1-5, 2015

7. J. D. Jia, "Combustion Feature Enhancement in Diesel Engine based on Cross-Wavelet Transform and Teager Energy Operator," Automotive Engineering, Vol. 36, No. 2, pp. 164-167, 2014

8. Z. Meng and Z. Liang, "Fault Diagnosis Method for Single Channel Rotating Machinery based on EMMD and BSS," Chinese Journal of Scientific Instrument, Vol. 34, No. 3, pp. 635-642, 2013

9. C. Zhang, "Fault Diagnosis Research of Rotating Machinery based on Adaptive Processing of Vibration Signal," Xidian University, 2012

10. R. X. Chen, B. P. Tang, and J. H. Ma, "Adaptive De-Noising Method based on Ensemble Empirical Mode Decomposition for Vibration Signal," Journal of Vibration and Shock, Vol. 31, No. 15, pp. 82-86, 2012

11. G. B. Guang, "Researeh on Method of Rotating Maehinery Fault Diagnosis based on Manifold Learning," Central South University, 2010

12. C. X. Li, G. Guo, Y. Wang, L. H. Zhao, and C. Zhang, "Ballistic Group Target Clustering Recognition Method based on Feature Sensitivity," Journal of Equipment Academy, Vol. 26, No. 3, pp. 52-57, 2015 